\title{
Pratiques
}

Linguistique, littérature, didactique

$169-170 \mid 2016$

Enseignement/apprentissage de la langue, des textes et des discours. 40\&nbspans de Pratiques

\section{L'enseignement du verbe à l'école. Des tensions entre enseignants et élèves de CM2}

Teaching Verbs in School. Tensions between Teachers and Students of CM2

\section{Patrice Gourdet et Marie-Noëlle Roubaud}

\section{(2) OpenEdition \\ Journals}

Édition électronique

URL : http://journals.openedition.org/pratiques/3059

DOI : $10.4000 /$ pratiques.3059

ISSN : 2425-2042

Éditeur

Centre de recherche sur les médiations (CREM)

Référence électronique

Patrice Gourdet et Marie-Noëlle Roubaud, « L'enseignement du verbe à l'école. Des tensions entre enseignants et élèves de CM2 », Pratiques [En ligne], 169-170 | 2016, mis en ligne le 30 juin 2016, consulté le 10 décembre 2020. URL : http://journals.openedition.org/pratiques/3059; DOI : https:// doi.org/10.4000/pratiques.3059

Ce document a été généré automatiquement le 10 décembre 2020.

(c) Tous droits réservés 


\section{L'enseignement du verbe à l'école. Des tensions entre enseignants et élèves de CM2}

Teaching Verbs in School. Tensions between Teachers and Students of CM2

Patrice Gourdet et Marie-Noëlle Roubaud

\section{Introduction}

1 L'enseignement du verbe à l'école cristallise les insatisfactions professionnelles. Le rapport de l'Inspection générale de l'éducation nationale (IGEN, 2013), sur la mise en œuvre des programmes de l'école primaire de 2008, indique que pour les enseignants des classes d'élèves de 8 à 11 ans : «En grammaire, c'est le travail autour du verbe qui occasionne le plus de difficultés (p. 19) » et que « l'obnubilation sur la conjugaison tient à la visibilité à l'écrit avec l'orthographe grammaticale (p. 20)». Cette centration sur la conjugaison est rappelée dans le rapport ministériel sur « L'étude et l'enseignement de la langue » (MEN ${ }^{1}, 2013$, p. 6) qui rend compte des pratiques réelles en étude de la langue d'enseignants de collège et de CM2 (élèves de 10-11 ans): les entrainements de conjugaison occupent $86 \%$ des activités des enseignants interrogés de CM2.

Ce même rapport révèle que 70 \% des enseignants de CM2 déclarent n'éprouver aucune difficulté à construire des progressions en grammaire (p. 7), l'enseignement de la langue présentant pour eux un caractère logique et rigoureux plutôt simple à mettre en œuvre : activités d'entrainement et évaluation fréquente passant par des exercices d'application. Néanmoins, ils perçoivent une certaine forme d'inefficacité de cet enseignement chronophage (des conjugaisons entre autres) qui ne se traduit pas par des acquis solides à l'entrée au collège (p.15). La lecture de ces deux documents ministériels montre que l'enseignement du verbe à l'école est le lieu de tensions.

3 Pour mieux les analyser, nous avons questionné par écrit 72 enseignants titulaires de cycle 3 sur leurs définitions du verbe et avons mis en perspective leurs réponses avec 
celles de 491 élèves de $\mathrm{CM} 2$, interrogés dans le cadre d'une recherche précédente à partir du même protocole ${ }^{2}$. Une grille de lecture a permis des comparaisons entre les réponses des deux groupes. Même si «les pratiques enseignantes aujourd'hui dominantes relèvent d'une pédagogie invisible ${ }^{3}$, quelle que soit la composition des classes» (Bautier, 2006, p. 109), les représentations des élèves sur le verbe laissent entrevoir les indices qu'ils prélèvent, la façon dont ils les interprètent et les savoirs effectifs sur lesquels ils s'appuient ${ }^{4}$. Il apparait alors « un écart massif entre ce que les élèves savent et ce qu'on croit qu'ils savent» (Brissaud \& Cogis, 2002, p. 39), accentuant les contradictions entre enseignement et apprentissage.

Notre réflexion linguistique construite sur une analyse méthodique d'une réalité langagière enrichit et donne du sens à la recherche didactique. En retour, les données sur l'enseignement nourrissent la réflexion linguistique sur le système de la langue. L'enjeu de nos recherches est bien de revitaliser l'enseignement du verbe à l'école, d'offrir un état des lieux précis pour construire des perspectives didactiques concrètes et des pistes pédagogiques pour son apprentissage.

\section{Les données observées}

\subsection{La méthodologie}

5 Notre travail sur le verbe s'inscrit dans une dynamique portée par le groupe Episteverb ${ }^{5}$. Un des axes de notre recherche porte sur l'analyse de questionnaires. Pour l'étude présentée ici, la question suivante avait été posée aux professeurs des écoles : «Vous êtes enseignant(e) en cycle 3 et vous devez expliquer le verbe, comment définiriez-vous cette notion? ». Cette question ouverte renvoie d'un côté à un positionnement professionnel et de l'autre à une demande d'explication qui doit entrainer l'utilisation de propriétés et permettre ainsi d'analyser les connaissances personnelles de l'adulte interrogé (Gourdet, 2010b, p. 27). Pour les élèves, la question était plus directe « Qu'est-ce qu'un verbe ? », elle se situe plus dans une demande de "restitution de savoir» qui reste une pratique répandue à l'école mais très souvent sous une modalité plutôt orale et collective (Gourdet \& Cogis, 2014, p.50). Le recueil s'effectue sur une fiche standardisée d'un format spécifique (un tiers d'A4) :

Document 1. Reproduction de la réponse d'un enseignant de cycle 3 (novembre 2014)

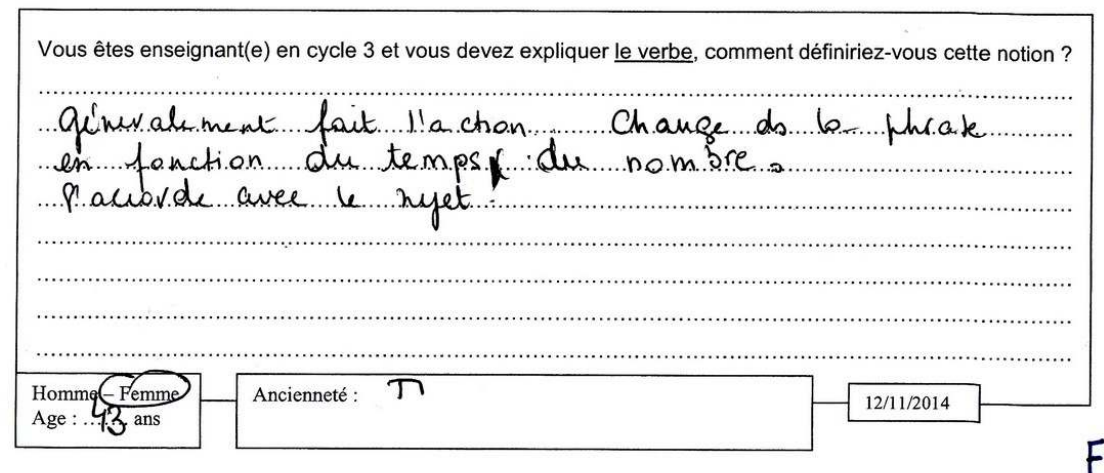




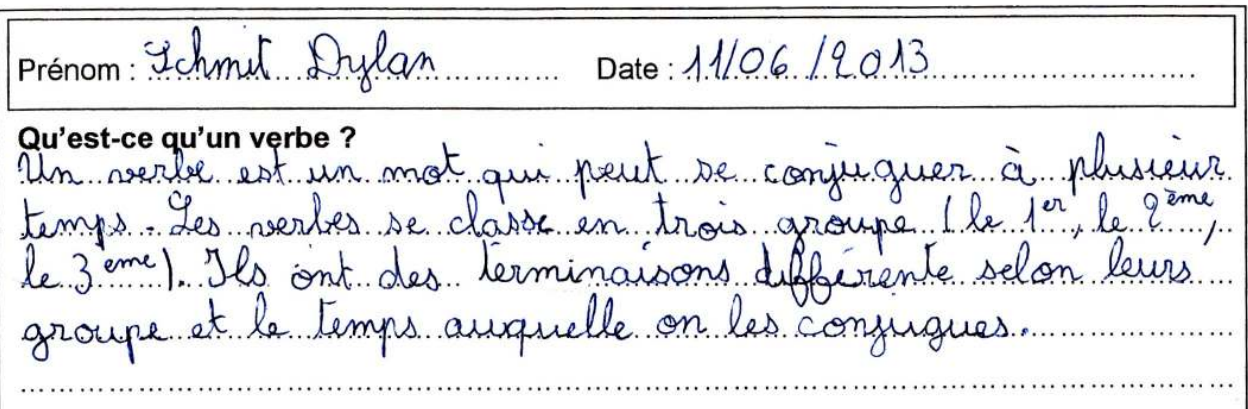

6 Tous les questionnaires ont été anonymisés : les enseignants ont été codés par une lettre (H pour homme, F pour femme) et un nombre (cf. supra : F21), les élèves : par un numéro d'ordre, par la classe et par un code pour l'école.

7 Tant pour les enseignants que pour les élèves, la procédure d'analyse des écrits proposés est identique. Nous cherchons à repérer les propriétés en codant les réponses par le biais d'une grille unique (cf. Annexe 1) qui répertorie un ensemble d'items regroupés en fonction de différentes entrées : sémantique, morphologique, syntaxique, phonologique, lexicale, textuelle. L'entrée sémantique peut renvoyer au lien du verbe avec l'opposition action-état, à la notion de procès ou au sens même de la phrase. Pour la morphologie, nous retrouvons l'idée de variation, de décomposition en radical-terminaison, de changement $d u$ verbe en fonction de la personne ou du temps. L'entrée syntaxique renvoie à l'accord avec le sujet, au lien avec les pronoms de conjugaison, au fait que le verbe est le noyau de la phrase, à l'encadrement par la négation, etc. Cette grille constitue un filtre d'analyse linguistique, sur les plans synchronique et diachronique ${ }^{6}$, utilisé pour qualifier les propriétés avancées dans les réponses et pour les quantifier. Par exemple, pour les deux propositions ci-dessous, le traitement des fiches permet de dégager les éléments suivants :

Document 3. Analyse de la réponse d'un enseignant et d'un élève de CM2 selon la grille

\begin{tabular}{|c|c|}
\hline Réponse de l'enseignant - F21 & Réponse de l'élève de CM2 - Dylan \\
\hline 18 mots (en comptant «s'accorde » comme 1 ) & 37 mots (sans compter les nombres) \\
\hline $\begin{array}{l}4 \text { items recensés (SEM1 - MO7 - MO4 - SYN4): } \\
\text { - une entrée sémantique qui fait référence à l'action ; } \\
\text { - deux items morphologiques avec l'idée d'un mot qui } \\
\text { varie en nombre et la variation en fonction du temps; } \\
\text { - une entrée syntaxique avec l'accord avec le sujet. }\end{array}$ & $\begin{array}{l}3 \text { propriétés morphologiques } \\
\text { recensées (MO7-MO2-MO5): } \\
\text { - la conjugaison en fonction du } \\
\text { temps; } \\
\text { - la classification des verbes (aspect } \\
\text { formel des groupes); } \\
\text { - la référence aux terminaisons. }\end{array}$ \\
\hline
\end{tabular}




\subsection{Les enseignants interrogés}

8 Le recueil s'est effectué le 12 novembre 2014 lors d'une conférence sur le verbe au cycle 3. Tous les enseignants (au nombre de 72) appartenaient à une même circonscription et avaient été conviés à un temps de formation continue.

Le questionnaire a été distribué à l'ouverture sans que les participants en aient été informés. Le contexte de la recherche a été expliqué avec la garantie de l'anonymat. Les enseignants ont eu 5 minutes pour répondre puis la fiche a été immédiatement récupérée afin d'éviter un retour sur ce premier jet durant la conférence.

1072 enseignants du premier degré titulaires composent cet échantillon, avec $85 \%$ de femmes (au niveau national, la féminisation dans le premier degré public est de $82,6 \%^{7}$ ), d'âge moyen de 33 ans (41,7 ans sur le plan national). Ils exercent en banlieue parisienne dans une circonscription urbaine. L'ancienneté moyenne dans l'enseignement est de 9 ans et 4 mois : ces enseignants sont dans le premier tiers de leur parcours professionnel.

11 Il n'y a eu aucune non-réponse et l'ensemble des écrits proposés représente 2110 mots, ce qui fait une moyenne de 29,3 mots par enseignant. Après codage des propriétés, nous en recensons 290 d'où une moyenne de 4,028 propriétés par réponse ${ }^{8}$.

\subsection{Les élèves interrogés}

12 Le recueil s'est effectué sur une dizaine de jours durant le mois de juin 2013. Les 491 élèves de CM2 sollicités avaient déjà été interrogés selon le même protocole quand ils étaient en CE2 dans le cadre d'un suivi de cohorte (Gourdet, 2014).

13 Le questionnaire a été présenté à 32 classes au sein de 15 écoles primaires sans que les élèves en aient été informés. Comme pour les enseignants, les élèves avaient 5 minutes pour répondre à la question posée, sans avoir pu anticiper la réponse qu'ils allaient donner.

14 Ces élèves sont issus d'un milieu urbain avec un éventail assez large des types d'école (2 sont dans un dispositif ÉCLAIR, 7 en RRS9 et 6 sont sans « étiquette »). Cette hétérogénéité confère au corpus un mixage social qui offre une forme de représentativité des catégories socioprofessionnelles des élèves.

15 Comme dans le cas des enseignants, tous les élèves ont répondu : seul un élève a eu besoin de l'aide d'un adulte à qui il a dicté sa définition. L'ensemble des réponses représente 13703 mots, ce qui donne une moyenne de 27,9 mots par élève. Nous recensons 1185 propriétés, c'est-à-dire 2,413 propriétés par réponse.

\subsection{L'extension du corpus}

16 Afin de mettre en contexte d'un point de vue institutionnel les réponses aux questionnaires, nous nous sommes référés aux Horaires et programmes d'enseignement de l'école primaire (MEN, 2008) qui fixe le cadre prescriptif en vigueur au moment de l'enquête. Un complément à ce texte a été édité dans le Bulletin officiel $\mathrm{n}^{\circ} 25$ du 19 juin 2014, sous le titre Recommandations pour la mise en cuvre de programmes (MEN, 2014). Deux autres documents ministériels faisant le bilan de la mise en œuvre des programmes de 
2008 ont été consultés : l'un pour l'école primaire (IGEN, 2013) et l'autre pour le collège incluant une réflexion sur l'articulation CM2-6 ${ }^{e}$ (MEN, 2013).

Des objets didactiques ont également servi à notre analyse : manuels, cahiers de règles, affichages, fournissant aux élèves un "environnement grammatical explicite » et à

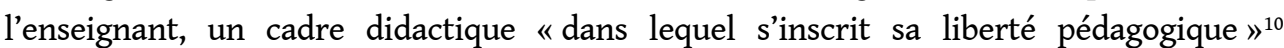
(Gourdet, 2010a, p. 58). C'est dans la relation entre les attentes officielles, le cadre didactique et les réponses des élèves qu'apparaissent en filigrane des tensions que nous allons essayer de préciser.

\subsection{Des données invisibles}

Sous toutes ces réponses se cachent deux évidences qui transparaissent dans les questionnaires, sans qu'il soit possible de les mesurer précisément.

19 La première est que l'enseignement du verbe est tributaire des propres connaissances linguistiques de l'enseignant (Elalouf, 2008; Eshkol, 2005 ; Gourdet, 2010a ; Roubaud \& Touchard, 2004) et de sa réflexion métalinguistique (Grossmann, 1998). Comme l'écrit D. Manesse (2013, p. 236), beaucoup d'enseignants ne font que « juxtaposer des contenus nouveaux à des connaissances construites depuis longtemps, et non intégrer ces deux types de connaissances ».

20 La seconde est que le savoir grammatical des élèves est «le résultat d'un processus d'assimilation et de réélaboration » (Othenin-Girard \& de Weck, 1988, p. 54). Il faut être conscient que le questionnaire fige le savoir à un moment $\mathrm{T}$ de l'apprentissage des élèves. Il n'en demeure pas moins que son analyse offre des pistes intéressantes pour comprendre l'enseignement du verbe à l'école.

\section{Aperçu des tensions au travers des questionnaires}

\subsection{Des écarts sur le choix des propriétés du verbe}

21 La codification des réponses, selon la grille de lecture (cf. Annexe 1), permet de quantifier le nombre d'enseignants et d'élèves qui actualisent au moins un des items appartenant aux différentes entrées. Nous constatons un décalage non négligeable entre ces deux groupes qui ne valorisent pas les mêmes entrées pour définir le verbe : 
Document 4. Proportion d'enseignants et d'élèves de CM2 utilisant au moins une propriété (ou item) par entrée

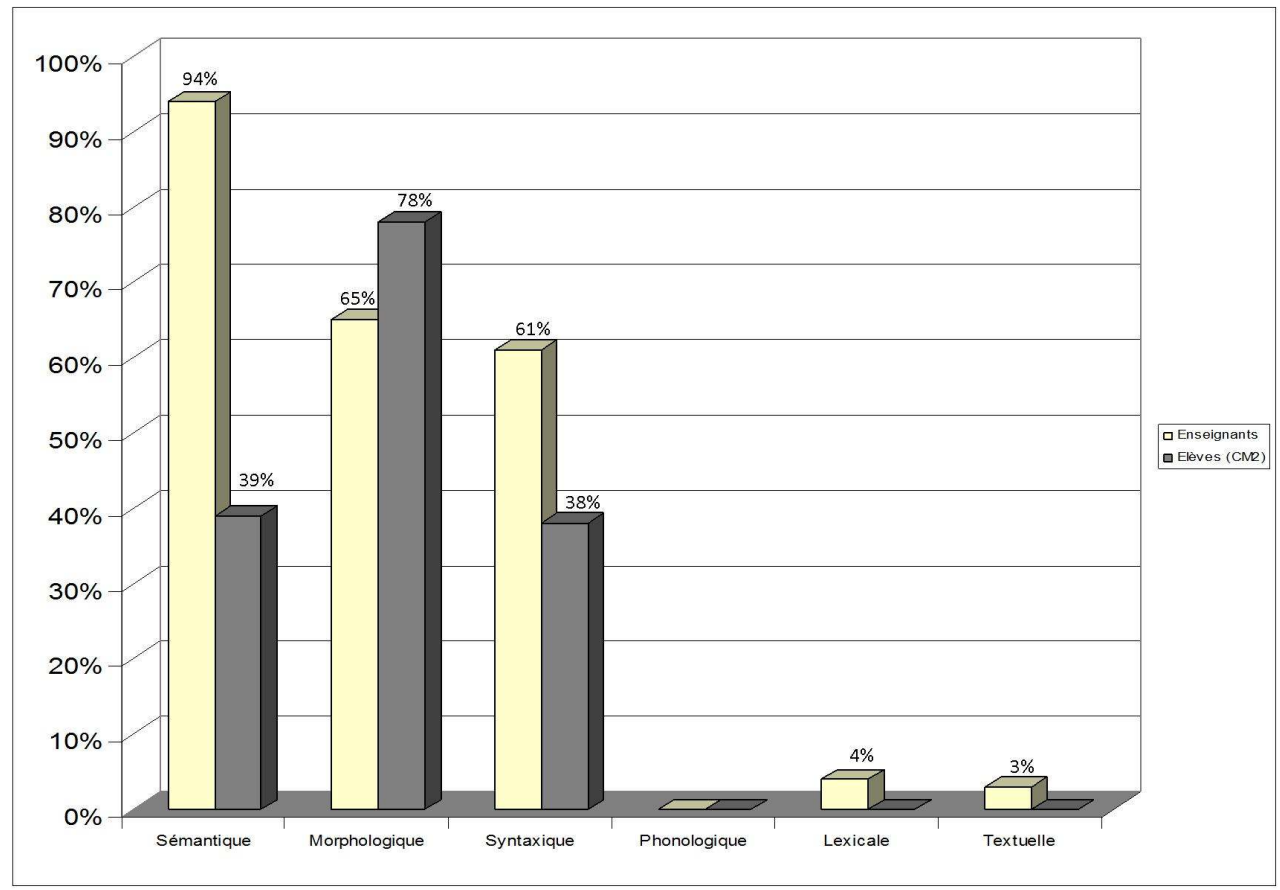

La quasi-totalité des enseignants mettent en avant l'entrée sémantique alors que les entrées morphologiques et syntaxiques sont citées par les deux tiers du groupe. De leur côté les élèves de CM2 sont surtout préoccupés par les questions de morphologie (78\%), les entrées sémantiques et syntaxiques n'étant référencées que par un tiers du groupe. Ce décalage montre que les préoccupations ne sont pas tout à fait les mêmes entre ceux qui enseignent et ceux qui apprennent.

Le grand absent pour ces deux groupes est l'entrée phonologique qui n'est jamais citée, ce qui confirme les recherches que nous avons menées sur d'autres corpus (Gourdet, 2014, p. 234). Cette absence de l'aspect phonologique semble indiquer une non prise en compte pédagogique du lien entre l'oral et l'écrit, pourtant mis en avant sur le plan linguistique depuis près d'un demi-siècle (Dubois, 1967; Pinchon \& Couté, 1981; Le Goffic, 1997; Meleuc \& Fauchart, 1999; Blanche-Benveniste, 2005). S'appuyer sur les formes orales permet de mettre en évidence les différentes bases verbales, d'opérer des regroupements entre verbes et d'observer des régularités morphologiques. Oraliser les désinences aide l'élève à réduire les possibilités graphiques au moment de l'écriture (Pinchon \& Couté, 1981, p. 47) et à diminuer le doute orthographique. Ainsi la désinence [õ] n'a en fait que deux actualisations écrites possibles: -ons ou -ont. L'absence de l'entrée phonologique dans l'ensemble des réponses est d'autant plus fâcheuse que très souvent les erreurs des élèves quand ils ont à écrire des formes verbales sont l'illustration du décalage entre les formes que les élèves ont oralisées et le système de l'écrit (Cappeau \& Roubaud, 2005, p. 93). Il semble que le travail pédagogique sur le rapport oral-écrit et les régularités des désinences de personne et de nombre (la conjugaison horizontale), qui sont maintenant préconisés par le Bulletin Officiel du mois de juin 2014 (MEN, 2014), ne soient pas encore une réalité dans les classes. Il est dommageable pour l'enseignement (et en retour pour l'apprentissage) que de telles recherches restent le plus souvent méconnues des enseignants. 
l'idée d'action (SEM1), semble incontournable alors que seulement $39 \%$ des élèves de CM2 utilisent cette entrée ${ }^{11}$ (192 élèves sur 491) dont 129 réfèrent à l'action et 63 citent la relation entre le temps de la phrase et le verbe (SEM3) ou le lien avec le sens de la phrase (SEM6) :

Document 5. Répartition des propriétés sémantiques chez les enseignants et les élèves de CM2

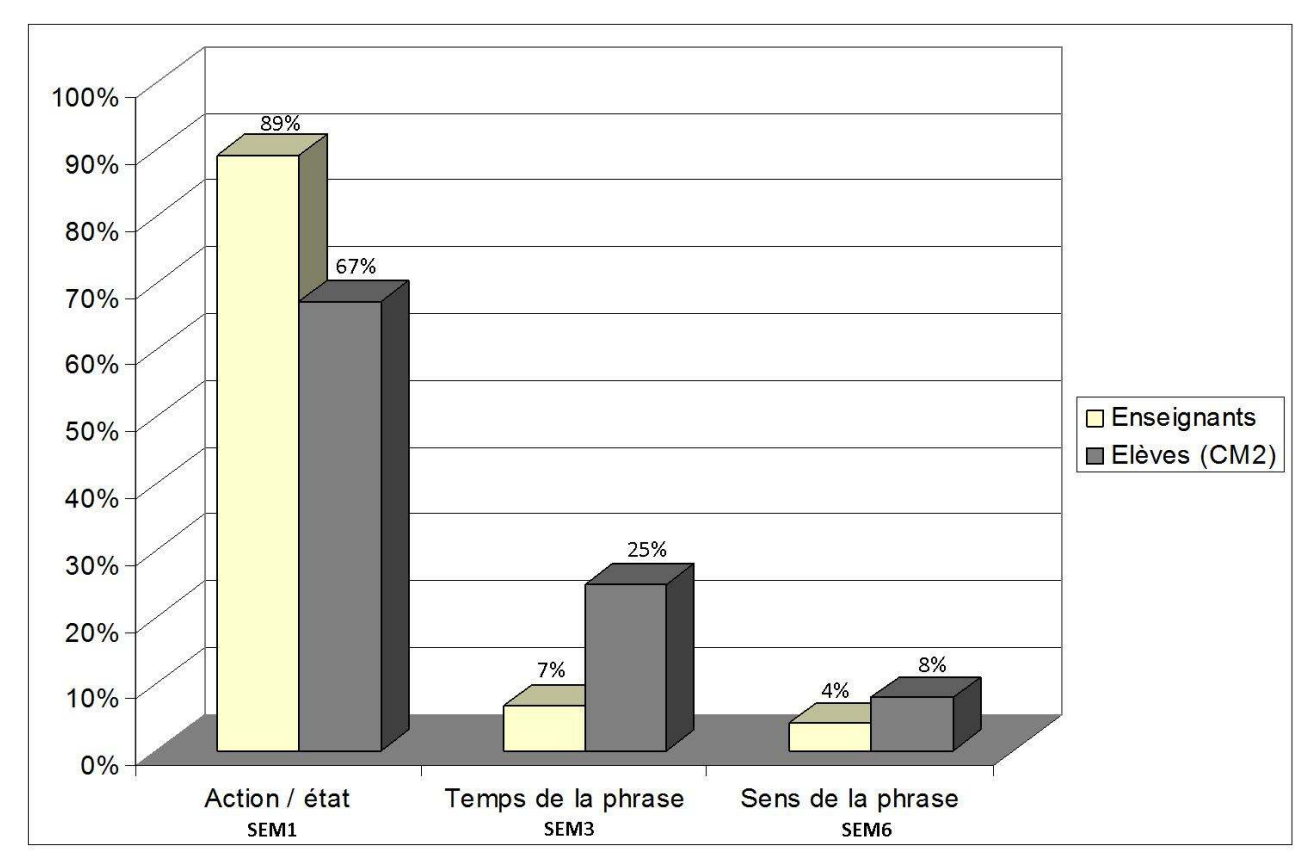

$89 \%$ des enseignants qui utilisent une entrée sémantique se réfèrent à l'action. La réponse de F41 est typique: Le verbe est un mot qui indique l'action de la phrase. On peut le mimer. D'autres enseignants avancent la dichotomie action/état afin de présenter un système simple et cohérent à deux ensembles. Mais l'enseignante F5 semble gênée par cette approche dichotomique : Je le ferais définir par les élèves, par un travail de déduction, en les faisant mimer. Puis, je les amènerais progressivement à les classer : verbes d'action... (courir, pousser, gémir etc.), verbes d'état et les autres « savoir, connaitre, penser... Je les définirais comme le mot au statut le plus instable de la phrase. La création d'une troisième famille "les autres " interroge l'entrée scolaire action/état qui ne semble pas adaptée sur le plan pédagogique pour appréhender la totalité des verbes. La question récurrente posée aux linguistes et aux didacticiens peut se résumer ainsi : ce passage par une définition du verbe sur le plan sémantique, focalisée sur l'idée d'action, que les jeunes élèves transforment en mouvement, est-il incontournable? Ce passage de la représentation du verbe par l'action constitue-t-il un palier cognitif $^{12}$ (Tisset, 2004, p. 35)? De plus, peut-on réduire la définition du verbe, sa conceptualisation, au simple fait de le mimer? Cette entrée par l'action est un savoir scolaire, institutionnalisé par les manuels scolaires pour définir le verbe, qui semble traverser le temps (cf. ces extraits de manuels de 1925 et de 2013) : 
Document 6. Première règle sur le verbe dans Cours de Langue Française, cours moyen, Hachette (1925, p. 135)

I7I. Le verbe. - Le verbe est un mot qui sert à exprimer une action ou un état. Ex. : Le père Bernard balayait le dallage de la salle commune (action); le père Bernard était un grand vieil homme (état).

Document 7. Première règle sur le verbe dans Réussir son entrée en grammaire au CE1, Retz (2013, p. 28)

\section{Le verbe}

Un verbe est un mot qui, le plus souvent, indique une action.

Exemple : Les enfants mangent. Le bébé dort.

Quand le verbe n'est pas dans une phrase, il est à l'infinitif : couper, manger, casser, dormir.

C'est aussi la forme que l'on utilise pour le nommer (par exemple dans le dictionnaire).

$\mathrm{Au} v \mathrm{vu}$ du pourcentage de réponses concernant les deux groupes, nous constatons une déperdition du savoir enseigné construit sur des connaissances partagées par les enseignants (savoir polarisé autour du fait que le verbe est l'action de la phrase). Une confusion s'installe chez les élèves qui associent fréquemment l'action à un déplacement physique, d'où des difficultés éventuelles à identifier penser, dormir, attendre comme des verbes. Des noms au sémantisme fort peuvent également indiquer des actions et être repérés comme des verbes : arrivée, départ, sortie (Riegel, Pellat \& Rioul, 1994, p. 244 ${ }^{13}$ ), cueillette, floraison (Tisset, 1998, p. 10), lecture (Lepoire-Duc \& Ulma, 2013, p. 241). Les élèves en recherche de sens sont déroutés parfois par l'interprétation du mot "action " comme l'indique l'explication de cet élève de 6 ans : « magicien c'est un verbe car il fait de la magie » (Gomila \& Roubaud, 2013, p. 38).

L'entrée sémantique ne peut donc être réduite au couple action versus état car ce savoir scolaire est inadapté pour comprendre et définir le verbe. Le terme même d'action questionne les linguistes : pour D. Leeman (2013), le terme "action» est à mettre en relation avec «(verbe à la) voix active ", par opposition à la voix passive, ce terme n'est donc pas à prendre de manière sémantique, il s'agirait en fait de syntaxe ${ }^{14}$.

La question du rapport sémantique au temps (SEM3) et du lien entre le verbe et le sens de la phrase (SEM6) sont, proportionnellement, surtout avancés par les élèves comme l'illustrent ces deux réponses: Un verbe peut servir à changer le temps d'une phrase (élève 16). Et Un verbe est un mots con peuts conjugai avec plusieur temps présen, passé, futur et ca donne un cense à la phrase (élève 34). C'est bien le rapport entre le verbe et la phrase avec la recherche de sens qui est mis en avant par ces quelques élèves, la phrase étant la dimension syntaxique ultime prônée par les manuels. 


\subsection{Des divergences dans l'utilisation des propriétés morphologiques}

L'analyse des données montre que l'entrée morphologique est bien représentée: la morphologie est un savoir instruit par l'école, plus souvent convoqué par les élèves que par les maitres ( $78 \%$ des réponses vs $65 \%$ ). L'enquête ministérielle (MEN, 2013) le confirme puisque $86,2 \%$ des maitres de CM2 indiquent entrainer leurs élèves à conjuguer et le Ministère déplore que " cette activité se résume trop souvent à des récitations de tableaux de conjugaison, non à un enseignement raisonné de la morphologie verbale et du système des temps (p. 12) ».

Deux tiers des enseignants (47 sur 72) et trois quarts des élèves (386 sur 491) utilisent une des propriétés morphologiques référencées dans notre grille d'analyse :

Document 8. Répartition des propriétés morphologiques chez les enseignants et les élèves de CM2

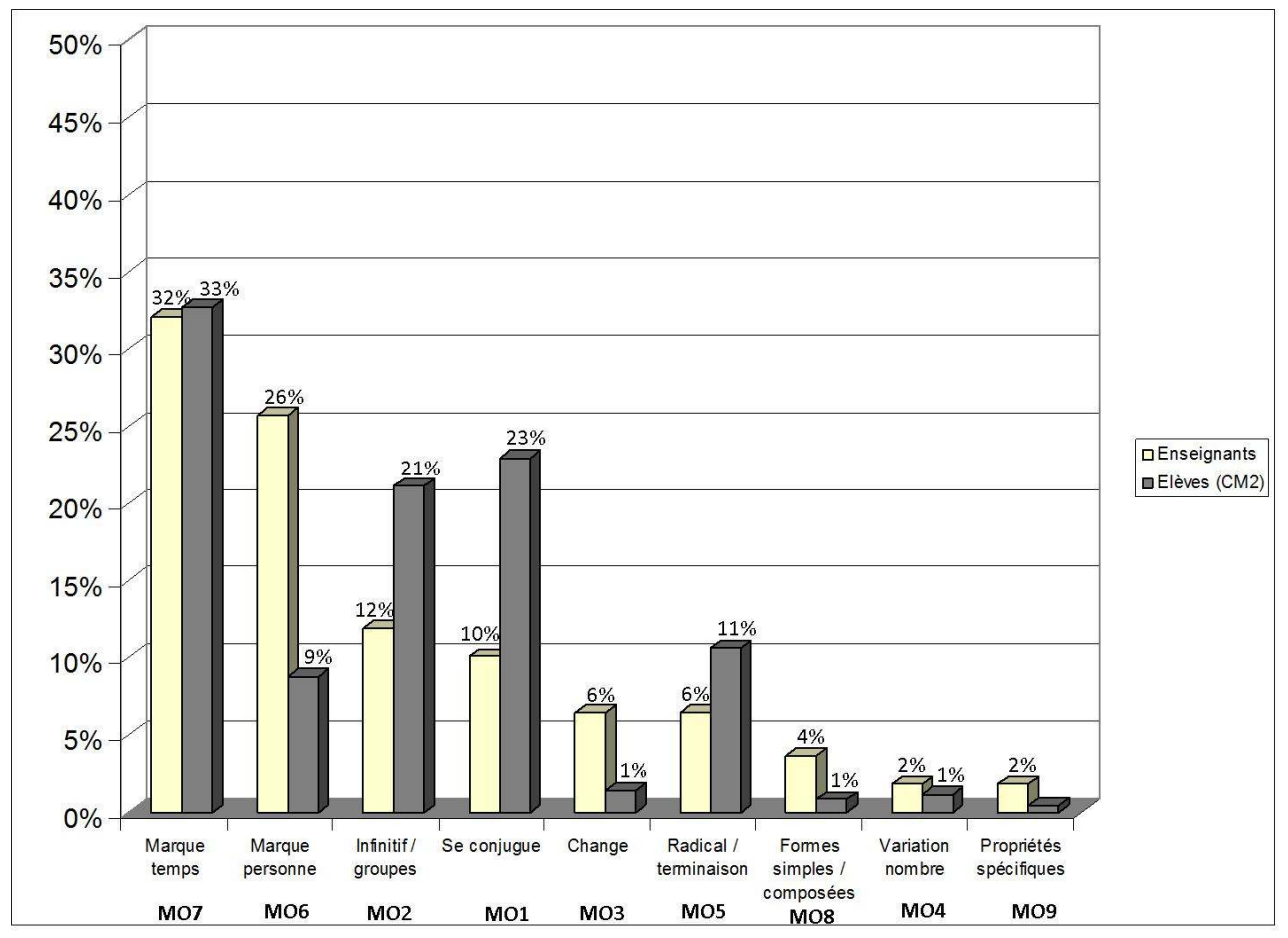

Mise à part la question de la variation des verbes en fonction du temps (MO7) citée par un tiers des deux groupes, nous constatons de nouveau des divergences fortes entre les enseignants et les élèves qui ne valorisent pas les mêmes propriétés quand ils font référence à la morphologie.

Les enseignants ont perçu les deux désinences, celle du temps (32\%) et celle liée au sujet (26\%) alors que seulement $9 \%$ des élèves indiquent la marque de personne (MO6). Cette dernière est peu identifiée dans les écrits, étant le plus souvent non sonore et problématique en orthographe. Il semble que les élèves amalgament la terminaison à la marque de temps souvent sonore sachant que là aussi le travail de décomposition et d'analyse des désinences reste une innovation pédagogique à inventer (Gourdet, 2013a, p. 53). La marque de personne est un objet d'apprentissage à aborder en isolant cet élément, obligeant alors à démonter la « terminaison » et à se sortir des simples tableaux 
de conjugaison. Comme le souligne M. Lusetti (2008), « Du point de vue morphologique, il est nécessaire de désarticuler les différents éléments de la désinence pour en mesurer la complexité, le caractère composite et hétérogène (p.110)", ce qui aurait l'avantage de mettre en avant la variation en nombre, quasiment absente des réponses (MO4). Cette absence pourrait souligner le fait que la variation en nombre, chez les élèves, serait réservée au nom. Cette préconisation didactique de distinguer « les marques liées au sujet et les marques liées au temps» est reprise dans le texte ministériel sur les recommandations pour la mise en œuvre des programmes (MEN, 2014).

D'autres différences sont perceptibles comme une sensibilité plus grande des élèves à l'aspect formel, marquée par des réponses mentionnant l'infinitif et les groupes (MO2), le radical et les terminaisons (MO5). Mais ces réponses, données dans un environnement scolaire (la classe), apparaissent souvent comme une " déclaration de règles ", les élèves reprenant le discours déclaratif des maitres d'une façon mécanique. C'est le cas de l'élève 136 qui écrit: Un verbe c'est quelque chose qui à un terminaison de plusieurs temps et il à trois groupe le $1^{\text {er }}$ groupe términaison er $2^{\text {ème }}$ groupe ir et $3^{\text {ème }}$ groupe tout se qui reste. À la lecture de cette réponse plutôt formelle, nous avons l'impression d'avoir une explication très incantatoire et peu opérationnelle qui nous amène à poser cette question : cet élève est-il capable d'identifier un verbe et de l'utiliser?

\subsection{Des propriétés syntaxiques différemment sollicitées}

Il n'y a que $61 \%$ d'enseignants (44 sur 72 ) et $38 \%$ d'élèves de CM2 (189 sur 491 ) qui définissent le verbe en utilisant une propriété syntaxique :

Document 9. Répartition des propriétés syntaxiques chez les enseignants et les élèves de CM2

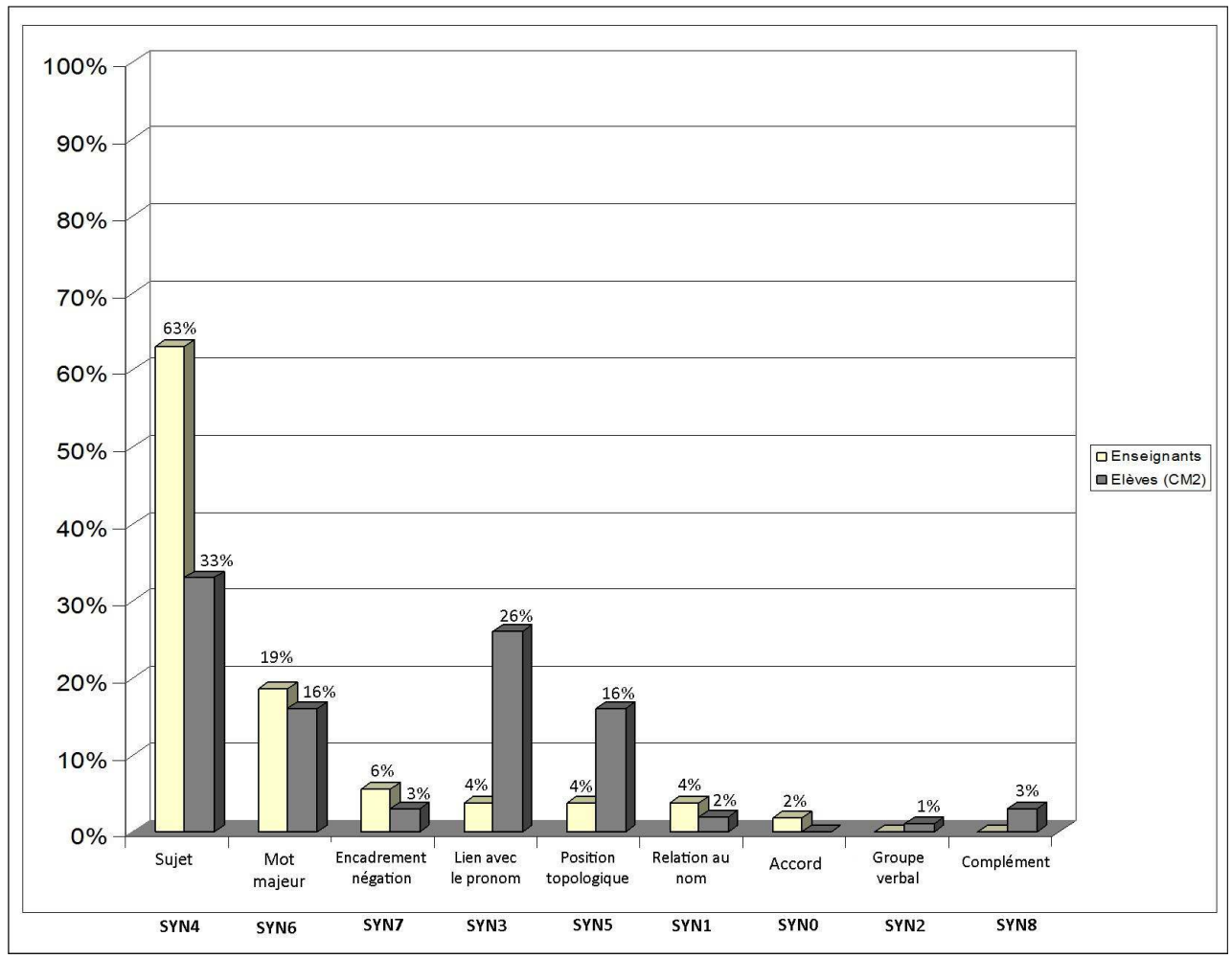



chez les maitres ( $63 \%$ vs $33 \%$ ). Pour les élèves, c'est avant tout dans le lien avec les pronoms de conjugaison ( $26 \%$ ) que le verbe est identifié (SYN3) alors que seulement $4 \%$ des enseignants le mentionnent. La mise en avant du lien du verbe avec le pronom (et pas avec le nom, cf. SYN1), indépendamment de la notion de sujet, tend à démontrer le poids des tableaux de conjugaison dans l'enseignement du verbe à l'école. Ce point est confirmé par leur prédominance dans les cahiers de règles (Gourdet, 2013b, p. 272) : on peut supposer que pour les élèves, ces tableaux occultent le fait que le pronom est un sujet au même titre que le nom. De plus, les règles sur le « sujet » sont très souvent isolées au sein de la grammaire et peu articulées avec la conjugaison du verbe, cloisonnant ainsi les propriétés du verbe. Il apparait que les élèves de CM2 s'enferment dans la relation pronom-verbe, négligeant alors celle de sujet-verbe. explication à l'indication sur l'ordre des mots (SYN5) alors que peu d'enseignants le mentionnent (seulement $4 \%$ ). Les élèves jugent souvent ce critère fiable: "le sujet est devant le verbe ".

Enfin, dans les grands absents, nous avons la non référence aux compléments avec la question du lien entre syntaxe et lexique : les compléments construits par le verbe. Cette dimension novatrice sur le plan pédagogique pourrait permettre de replacer la question des compléments dans une dynamique autour du verbe et des termes qu'il régit ${ }^{15}$ et de se sortir des débats archaïques concernant l'intérêt de l'étiquetage plus que centenaire des compléments d'objet directs ou indirects. Comme l'écrit M. Csécsy (1968), «les exercices de conjugaison opèrent dans la dimension paradigmatique, alors que, pour nous servir d'une langue, il faut pouvoir créer des associations syntagmatiques (p. 24) ». Si l'approche verticale renforce le lien entre les clitiques et le verbe, elle est très éloignée des situations d'écriture des élèves. Le verbe est donc à pratiquer en discours : il n'est pas qu'une unité isolée, c'est une unité pré-construite (utilisée en contexte) qui pourrait servir de modèle syntaxique lors de l'écriture ${ }^{16}$ et par là-même limiterait les erreurs orthographiques puisque morphologie et syntaxe seraient deux dimensions construites conjointement. B. Combettes (2009) propose la même piste de réflexion: "Il semble indispensable de s'interroger sur les constructions que l'on estimera nécessaire de présenter aux élèves ; c'est à partir de ces structures que devraient être élaborées les progressions, le travail sur les natures et sur les fonctions passant au second plan, comme une aide éventuelle dans le travail sur les énoncés, et non comme une fin en soi (p. 46)».

\subsection{Des entrées laissées pour compte}

En plus de l'entrée phonologique qui est absente (cf. supra doc. 3), 3 enseignants sur 72 et 6 élèves sur 491 mentionnent l'entrée lexicale qui se résume à indiquer que «le verbe est un mot » (LEX0) et quant à l'entrée textuelle, il n'y a que 2 enseignants et 1 élève qui s'y réfèrent. Ce peu de réponses aux deux entrées confirme ce qui vient d'être observé pour la syntaxe : le verbe est perçu comme un élément isolé (une unité isolable du langage), sans que la dimension textuelle soit présente. Comme l'écrit M. Lusetti (2008): "Comment, une fois l'objet découpé, rendre accessibles et ordonner les éléments les uns aux autres? (p. 118)». Le savoir partiel et isolé des autres connaissances n'est pas efficient : on ne peut pas séparer les propriétés, elles font toutes parties intégrantes du verbe avec plus ou moins de force selon l'énoncé produit. 
La fréquence des mots (comptage en fonction du nombre total de mots dans les questionnaires) confirme les entrées repérées et les propriétés valorisées par les deux groupes. Alors que les enseignants pensent phrase-verbe-action, les élèves pensent verbeconjugaison-mot. Là encore un décalage entre enseignement et apprentissage apparait :

Document 10. Podium des mots récurrents dans les réponses des enseignants et des élèves de CM2

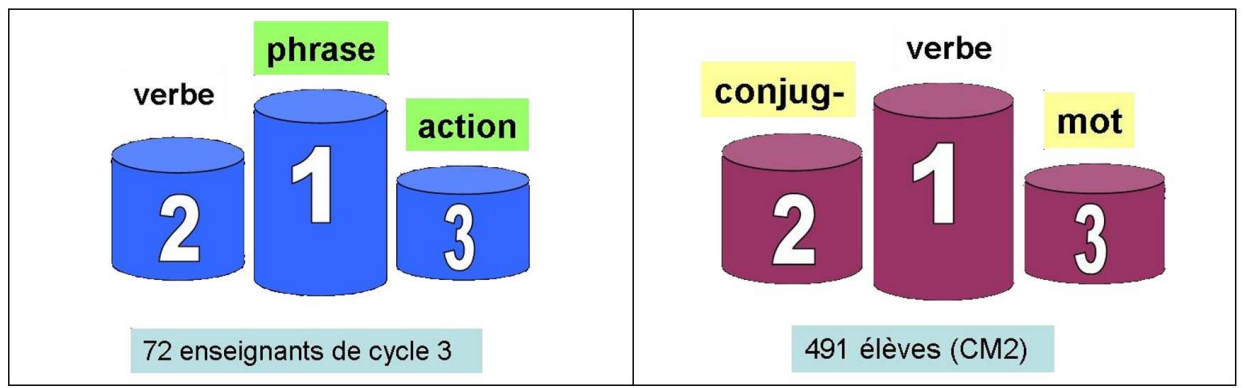

Il en ressort que l'enseignement du verbe porte sur le verbe dans la phrase, c'est-à-dire comment il s'écrit et non pas dans le texte, c'est-à-dire comment il s'emploie, ce que la fréquence des mots chez les élèves corrobore (cf. doc. 10). Les contenus abordés à l'école en grammaire ne sont pas articulés avec les usages de la langue.

\section{Conclusion}

La comparaison des réponses des maitres et des élèves de $\mathrm{CM} 2$ montre que des décalages existent entre ce que les maitres enseignent, ce que les élèves retiennent et/ou comprennent, les deux groupes ne valorisant pas les mêmes propriétés, d'où des déperditions entre enseignement et apprentissage, des insatisfactions et des tensions.

Même si tous les maitres essaient de créer des automatismes (cf. les déclarations de règles des élèves), s'appuient sur des métaphores ${ }^{17}$ (le verbe est le noyau/le moteur de phrase) pour faire identifier le verbe, ce formatage conceptuel le réduit à sa conjugaison en favorisant une entrée sémantique réductrice (l'action) et en privilégiant le lien avec le pronom. Les maitres ne perçoivent pas la langue comme un système ${ }^{18}$ et n'envisagent pas le verbe comme un constructeur d'énoncés à l'articulation d'une relation au temps et au nom. Alors comment pourrait-il en être autrement pour les élèves ?

Comme l'écrit le Ministère (IGEN, 2013), «la recherche doit être encouragée voire soutenue sur des sujets prioritaires [...] trois relèvent de l'urgence : l'enseignement de l'oral, celui du vocabulaire [...] et celui de la conjugaison (p. 26)». Or la recherche apporte depuis longtemps des réponses : $C$. Tisset propose de réaliser « très régulièrement des tris de mots et des exercices de commutation oraux et écrits " afin de faire "verbaliser le changement de sens et les changements orthographiques (1998, p. 11)», de "s'appuyer sur les représentations des élèves et sur leur connaissance intuitive des régularités de conjugaisons pour bâtir une progression $(1999$, p. 5) ». M.-N. Roubaud \& M.-J. Moussu (2010) mettent en place une progression spiralaire et linéaire pour l'apprentissage du verbe par de jeunes élèves.

En ce début de $\mathrm{XXI}^{\mathrm{e}}$ siècle, il semble essentiel de réinterroger l'enseignement du verbe à la lumière des connaissances actuelles en prenant en compte, par exemple, le rapport 
oral-écrit, le contexte sémantique et syntaxique, le poids du syntagme au sein d'une approche qui hiérarchise et organise les différentes dimensions, les met en système tout en proposant des niveaux de formulation adaptés aux élèves.

Pour être plus efficace, il est indispensable de réduire ces décalages et ces tensions entre enseignement et apprentissage. Pour atteindre cet objectif, il faut penser une approche dynamique du verbe articulant les pratiques scolaires d'enseignement, les usages et les besoins langagiers des élèves. Nous pensons que cette « révolution » ne peut se réaliser sans une formation initiale et continue de qualité, étayée par des savoirs linguistiques et didactiques rénovés.

\section{BIBLIOGRAPHIE}

BAUTIER, É. (2006). « Le rôle des pratiques de maîtres dans les difficultés scolaires des élèves : une analyse de pratiques intégrant la dimension des difficultés socialement différenciées ». Recherche et formation 51, p. 105-118.

BERNSTEIN, B. (1975). Classe et pédagogies : visibles et invisibles. Paris : OCDE.

BLANCHE-BENVENISTE, C. (2005). « Structure et exploitation de la conjugaison des verbes en français contemporain ». Le Français aujourd'hui 148, p. 75-87.

BRISSAUD, C. \& COGIS, D. (2002). « La morphologie verbale écrite, ou ce qu'ils en savent en CM2 ». Lidil 25, p. 31-42.

CAPPEAU, P. \& ROUBAUD, M.-N. (2005). Enseigner les outils de la langue avec les productions d'élèves. Paris : Bordas.

CSÉCSY, M. (1968). De la linguistique à la pédagogie : le verbe français. Paris : Hachette/Larousse.

DUBoIs, J. (1967). Grammaire structurale du français. Le verbe. Paris : Larousse.

combettes, B. (2009). « Quelle(s) description(s) grammaticales (s) pour l'enseignement ? » Repères 39, p. 41-56.

ELALOUF, M.-L. (2008). « Des outils effectifs du futur enseignant à la convocation des savoirs linguistiques et réciproquement ». Diptyque 14, p. 103-117.

ESHкоL, I. (2005). « La construction du concept de “verbe" ». Diptyque 4, p. 17-36.

GOMILA, C. \& ROUBAUD, M.-N. (2013). « Le verbe au cours préparatoire : premières constructions du concept ». In : Avezard-Roger, C. \& Lavieu-Gwozdz, B. (éds), Le verbe : perspectives linguistiques et didactiques. Arras : Artois Presses Université, p. 31-45.

GOURDET, P. (2009). L'enseignement de la grammaire à l'école élémentaire : le cas du verbe en CE2. Thèse de doctorat, Université Paris Ouest - Nanterre-La Défense.

- (2010a). «La classe, un environnement grammatical particulier : description des éléments qui constituent l'environnement grammatical des élèves à l'école élémentaire française ». La lettre de l'Association internationale pour la Recherche en Didactique du Français 45-46, p. 58-65.

- (2010b). « Les savoirs enseignants sur la notion grammaticale de verbe ». Repères 42, p. 25-44. 
- (2013a). «L'enseignement du verbe à l'école élémentaire : perspectives linguistiques et pistes didactiques ». In : Avezard-Roger, C. \& Lavieu-Gwozdz, B. (éds), Le verbe : perspectives linguistiques et didactiques. Artois : Artois Presses Université, p. 47-58.

- (2013b). « Le "cahier de règles" à l'école élémentaire, outil institutionnel et référence grammaticale : le cas du verbe en CE2 ». In : Bertrand O. \& Schaffner, I. (dirs), Enseigner la grammaire. Palaiseau : Éditions de l'École polytechnique, p. 265-285.

- (2014). « Les explications linguistiques sur le verbe. Un suivi sur une année scolaire d'une cohorte d'élèves de CE2 ». In : Roubaud, M.-N. \& Sautot, J.-P. (dir.). Le verbe en friche. Approches linguistiques et didactiques. Berne : Peter Lang, p. 217-234.

GOURDET, P. \& COGIS, D. (2014). « Un verbe c'est quelque chose : emploi « profane » ou emploi “savant" du métalangage à l'école élémentaire ?» Le discours et la langue 6, 1, p. 47-62.

GROSSMANN, F. (1998). « Métalangue, manipulations, reformulation : trois outils pour réfléchir sur la langue ». In : Dolz, J. \& Meyer, J.-C. (éds), Activités métalangagières et enseignement du français. Berne : Peter Lang, p. 91-116.

LE GOFFIC, P. (1997). Les formes conjuguées du verbe français : oral et écrit. Paris : Ophrys.

LEPOIRE-DUC, S. \& ULMA, D. (2013). « Le verbe tel qu'il s'enseigne, le verbe tel qu'il se dit à l'école primaire : regards croisés d'enseignants et d'élèves sur le concept de verbe ». Diptyque 23, p. 231-252.

LUSETTI, M. (2008). « Le verbe pour commencer la grammaire au CE1 ». Recherches 48, p. 105-135.

MANESSE, D. (2013). « La grammaire scolaire et ses fantômes ». In : Bertrand, O. \& Schaffner, I. (dir.), Enseigner la grammaire. Palaiseau : Éditions de l'École polytechnique, p. 229-242.

Meleuc, S. \& FAUCHART, N. (1999). Didactique de la conjugaison. Le verbe " autrement ». Paris/ Toulouse : Bertrand Lacoste/CRDP Midi-Pyrénées.

OTHENIN-GIRARD, C. \& DE WECK, G. (1988). « Si le savoir grammatical ne reflétait pas seulement l'enseignement reçu ?» Le français aujourd'hui 83, p. 53-60.

PILLE, J.-P. \& PIÉRAUT-LE-BONNIEC, G. (1987). « Le dire et l'activité métalinguistique : le

développement de la notion de verbe ». In : Piéraut-Le-Bonniec, G., Connaître et le dire. Bruxelles : Pierre Mardaga.

PINCHON, J. \& COUTÉ, B. (1981). Le système verbal du français. Paris : Nathan.

QUET, F. \& DOUROJEANNI, D. (2004). « En cycle III, repérer le verbe ». In : Vargas, C. (dir.), Langue et études de la langue. Aix-en-Provence : Presses universitaires de Provence, p. 301-310.

RIEGEL, M., PELlAT, J.-C. \& Rioul, R. (1994). Grammaire méthodique du français. Paris : Presses universitaires de France.

ROUBAUD, M.-N. \& TOUCHARD, Y. (2004). « Vers la notion de verbe : de l'approche intuitive à la construction du savoir, vers sept ans ». In : Vargas, C. (dir.), Langue et études de la langue. Aix-enProvence : Presses universitaires de Provence, p. 257-267.

Roubaud, M.-N. \& GOMILA, C. (2014). « Premières justifications de la catégorie verbe au cours préparatoire. Un prototype en construction ». In : Roubaud, M.-N. \& Sautot, J.-P. (dir.). Le verbe en friche. Approches linguistiques et didactiques. Berne : Peter Lang, p. 177-192.

Roubaud, M.-N. \& Moussu, M.-J. (2010). « Pour une modélisation de l'enseignement de la grammaire au CE1 : l'exemple du verbe ». Repères 41, p. 71-90.

TESNIÈRE, L. (1959). Éléments de syntaxe structurale. Paris : Klincksieck. 
TISSET, C. (1998). « Surtout ne pas donner la définition du verbe ». BLÉ 91 - Bulletin de liaison des écoles de l'Essonne 23, p. 10-11.

- (1999). «Surtout renoncer aux tables de conjugaison ». BLÉ 91 - Bulletin de liaison des écoles de l'Essonne 25, p. 4-5.

- (2004). « Un jour fut le verbe ». Diptyque 4, p. 33-50.

\section{Documents ministériels (ordre chronologique)}

Ministère de l'Éducation nationale (2008). Bulletin Officiel, $\mathrm{n}^{\circ} 3$ du 19 juin 2008, Horaires et programmes d'enseignement de l'école primaire.

Inspection générale de l'éducation nationale. (2013). Bilan de la mise en cuvre des programmes issus de la réforme de l'école primaire de 2008. Rapport à monsieur le ministre de l'Éducation nationale, $\mathrm{n}^{\circ}$ 2013-066, juin 2013.

Ministère de l'Éducation nationale. (2013) L'étude et l'enseignement de la langue : Rapport sur la mise en œuvre du programme de français au collège, décembre 2013.

Ministère de l'Éducation nationale (2014). Bulletin Officiel, n 25 du 19 juin 2014, Recommandations pour la mise en cuvre des programmes.

\section{ANNEXES}

\section{Annexe 1}

\section{Qu'est-ce qu'un verbe?}

Grille de lecture pour analyser les propriétés mises en avant pour définir le verbe.

\section{Entrée sémantique (SEM)}

\begin{tabular}{|l|l|l|}
\hline SEM1 & $\begin{array}{l}\text { Référence à l'action ou à l'état sans référence au temps (le verbe est un mot qui exprime } \\
\text { l'action) }\end{array}$ \\
\hline SEM2 & Référence à une unité sémantique qui désigne un procès (processus) \\
\hline SEM3 & $\begin{array}{l}\text { Référence à un mot qui indique le temps du procès, qui permet de se repérer. } \\
\text { temps (il porte la marque du temps sur la phrase) }\end{array}$ \\
\hline SEM6 & $\begin{array}{l}\text { Référence au lien entre le verbe et le sens de la phrase, entrée sémantique dominante } \\
\text { (interaction forte avec SYN6) }\end{array}$ \\
\hline
\end{tabular}

\section{Entrée morphologique (M0)}

M01

Utilisation de l'expression se conjugue sans plus de précision (utilisation de mots tels que conjugaison également) 


\begin{tabular}{|l|l|l|}
\hline M02 & Approche utilisant l'infinitif et / ou la classification en groupes \\
\hline M03 & $\begin{array}{l}\text { Référence à un mot qui change, qui varie, qui se transforme (sans donner plus } \\
\text { d'informations) }\end{array}$ \\
\hline M04 & Référence à une variation singulier-pluriel, à une variation en nombre \\
\hline M05 & Référence à la notion de radicaux et/ou de terminaisons \\
\hline M06 & $\begin{array}{l}\text { Référence à un mot qui change en fonction d'une personne ou d'un sujet ou d'un } \\
\text { pronom personnel > ce code est étroitement lié aux codes SYN } 3 \text { et SYN } 4 \text { (voire SYN 1) }\end{array}$ \\
\hline M07 & $\begin{array}{l}\text { Référence à un mot qui change en fonction du temps (simple, composé) => référence } \\
\text { présent / futur) }\end{array}$ \\
\hline M08 & $\begin{array}{l}\text { Référence aux formes (simples ou composées) des verbes conjugués (avec intégration } \\
\text { éventuelle de l'auxiliaire dans la présentation en deux parties) }\end{array}$ \\
\hline M09 & Références et propriétés spécifiques : les modes / les verbes pronominaux, réfléchis... \\
\hline
\end{tabular}

\section{Entrée syntaxique (SYN)}

\begin{tabular}{|l|l|l|}
\hline SYN0 & $\begin{array}{l}\text { Référence à l'accord (sans plus de précisions) / Chaine syntaxique de plusieurs verbes } \\
\text { (sans plus de précisions) }\end{array}$ \\
\hline SYN1 & Référence à un lien entre le verbe et le(s) nom(s) \\
\hline SYN2 & Référence à la notion de groupe verbal (sans plus de précision) \\
\hline SYN3 & Référence à un lien avec un pronom de conjugaison \\
\hline SYN4 & $\begin{array}{l}\text { Référence à la fonction « sujet » en lien avec le verbe (s'accorde avec ou utilisation du } \\
\text { terme le sujet) }\end{array}$ \\
\hline SYN5 & $\begin{array}{l}\text { Référence à un positionnement topologique (verbe derrière le sujet par exemple) - La } \\
\text { phrase...) }\end{array}$ \\
\hline SYN6 & $\begin{array}{l}\text { Le verbe est considéré comme le mot majeur, le noyau de la phrase (son constituant } \\
\text { principal / le pivot) => lien direct avec la phrase (plus ou moins explicite) }\end{array}$ \\
\hline SYN7 & $\begin{array}{l}\text { L'encadrement du verbe conjugué par la négation } \\
\text { SYN8 }\end{array}$ & $\begin{array}{l}\text { Référence aux compléments (arguments) liés au verbe (utilisation des termes : } \\
\text { transitifs / intransitifs) }\end{array}$ \\
\hline
\end{tabular}


Entrée phonologique (PHON)

\begin{tabular}{|l|l|l|}
\hline PHON1 & $\begin{array}{l}\text { Référence aux variations sonores de certaines flexions temporelles ou certaines } \\
\text { flexions en personne => P4 et P5 (on entend) }\end{array}$ \\
\hline PHON2 & Référence au décalage entre l'oral et l'écrit (on n'entend pas) \\
\hline & PHON3 & D'autres phénomènes en lien avec l'oral \\
\hline
\end{tabular}

\section{Entrée lexicale (LEX)}

\begin{tabular}{|l|l|l|}
\hline & LEX0 & Référence au fait que le verbe est un mot \\
\hline & LEX & Entrée qui renvoie la définition et / ou le sens du verbe au dictionnaire \\
\hline & LEX2 & Référence à la classe grammaticale \\
\hline
\end{tabular}

\section{Entrée textuelle (TEXT)}

\begin{tabular}{|l|l|l|}
\hline & TEXT 1 & Lien entre le texte et le verbe (sans plus) \\
\hline & TEXT 2 & Lien entre le verbe, les événements / la chronologie et le texte \\
\hline
\end{tabular}

\section{Autres entrées}

\begin{tabular}{|l|l|l|}
\hline & NG1 & Explication non linguistique ou incompréhensible \\
\hline & NG2 & Non réponse \\
\hline & NG3 & Réponse générale (le verbe sert à bien parler le français) \\
\hline & NG4 & Utilisation d'une terminologie grammaticale non appropriée \\
\hline & EX & Une réponse n'utilisant que un ou des exemples \\
\hline
\end{tabular}

Grille actualisée - Patrice GOURDET \& Marie-Noëlle ROUBAUD - Janvier 2015

\section{NOTES}

1. $M E N=$ Ministère de l'Éducation Nationale.

2. P. Gourdet \& D. Cogis. (2014). Communication dans le cadre du 82e congrès de l'Acfas à Montréal (Canada), le 13 mai 2014 : «Un savoir en évolution: la notion de verbe. Suivi d'une cohorte d'élèves au cycle 3 (début $3 \mathrm{eP}$-fin $5 \mathrm{eP}$ )».

3. Référence à B. Bernstein (1975). 
4. C'est ce que nous avons déjà montré pour des élèves de 6 ans (Roubaud \& Gomila, 2014), de 7 ans (Roubaud \& Touchard, 2004 ; Roubaud \& Moussu, 2010) et de 8 ans (Gourdet, 2009). Cf. aussi F. Quet \& D. Dourojeanni (2004).

5. Episteverb est une association composée à l'origine de 7 enseignants-chercheurs (dont nousmêmes) de France et du Canada, regroupés autour de la problématique de l'enseignement et de l'apprentissage du verbe. Son objectif est de diffuser des travaux de recherche (publications, organisations de manifestations scientifiques...) et ainsi de proposer des pistes pour l'enseignement du verbe.

6. L'architecture de cette grille est issue d'un travail de thèse (Gourdet, 2009); la plupart des items demeurent pour permettre l'analyse de réponses d'élèves et d'enseignants sur plusieurs années. La grille a été actualisée en 2015 pour les entrées lexicales et textuelles.

7. Source ministérielle 2015, publication annuelle de la DEPP. En ligne: http:// cache.media.education.gouv.fr/file/2015/65/8/depp_rers_2015_personnels_454658.pdf.

8. Ce chiffre est plus élevé que lors de recherches précédentes : en 2010, il n'atteignait que 3,5 propriétés par réponse (Gourdet, 2010b, p. 31).

9. Le dispositif ÉCLAIR (Écoles, collèges et lycées pour l'ambition et la Réussite) et les RRS (Réseaux de réussite scolaire) étaient des dispositifs spécifiques pour aider les équipes pédagogiques. Ces classifications étaient liées aux difficultés sociales et scolaires des élèves. En 2015 , une nouvelle qualification est mise en place : les « REP + ».

10. Liberté affirmée dans les programmes de 2008 (MEN, 2008, p. 3).

11. À contrario, chez les élèves plus jeunes (avant 8 ans), l'entrée sémantique est prédominante (Roubaud \& Touchard, 2004 ; Gomila \& Roubaud, 2013). J.-P. Pille \& G. Piéraut-Le-Bonniec (1987) avaient déjà noté que vers 8 ans, les élèves abandonnaient progressivement les jugements sémantiques et pragmatiques au profit de critères morphologiques et syntaxiques.

12. C'est aussi ce que nous avons montré pour des élèves de 6 ans (Gomila \& Roubaud , 2013, p. 38).

13. Chez M. Riegel, J.-C Pellat \& R. Rioul (1994) l'angle syntaxique est privilégié et l'entrée sémantique est orientée vers l'idée que le verbe est un mot qui signifie le temps, l'action ne délimitant pas strictement la catégorie du verbe.

14. Conférence du 02 février 2013. En ligne: http://www.danielle-leeman.com/ Conference_institut_formation_pedagogique_lille.pdf.

15. Selon le concept de « valence verbale » de L. Tesnière (1959).

16. P. Gourdet \& D. Cogis (2014, p. 60) indiquent que ces « moules langagiers » seraient utiles aux élèves pour définir, exposer, expliciter, expliquer, sans oublier l'apprentissage de leur corollaire orthographique ».

17. Les métaphores sont nombreuses dans les questionnaires des enseignants.

18. C'est aussi ce qu'affirme le Ministère (IGEN, 2013, p. 25).

\section{RÉSUMÉS}

Enseigner le verbe à l'école n'est pas chose aisée. Un rapport ministériel auprès d'enseignants de $\mathrm{CM} 2$ et de collège montre que l'activité principale pour étudier la langue à l'école n'est pas la dictée $(67 \%)$ mais l'entrainement à conjuguer ( $86 \%)$. Il révèle également que la grande majorité des enseignants de CM2 déclarent n'éprouver aucune difficulté à construire l'enseignement de la 
langue, en particulier en grammaire. En parallèle, un rapport de l'Inspection générale de l'éducation nationale (2013), sur la mise en œuvre des programmes de 2008 à l'école primaire, souligne les difficultés des élèves à conjuguer le verbe. La lecture de ces deux textes ministériels souligne l'existence de tensions. Pour les identifier, nous avons, par écrit, interrogé d'un côté des enseignants titulaires de cycle 3 sur leurs définitions du verbe et de l'autre des élèves de CM2. La comparaison des réponses et des propriétés mises en avant pour définir le verbe permet de percevoir les décalages et les tensions entre d'un côté les connaissances et les pratiques en vigueur des enseignants et de l'autre les savoirs linguistiques. Notre contribution a pour objectif de revitaliser l'enseignement du verbe à l'école et d'offrir des perspectives didactiques concrètes.

Teaching verbs in school is not easy. A ministerial report from teachers in CM2 and in college shows that the main activity when studying language in school is not dictations (67\%) but training to conjugate verbs (86\%). It also reveals that most teachers in CM2 declare they have no difficulty in constructing teaching of the language, especially grammar. At the same time, a report from the General Inspectorate of Education (2013) on the implementation of the 2008 programs in elementary school, stresses the difficulties students are having in conjugating verbs. These two ministerial texts highlight the existing tensions. To identify these tensions, we asked, in writing, both holders of cycle 3 teachers and CM2 pupils about their definition of the verb. Comparing the answers and the properties put forward when defining the verb makes it possible to perceive the discrepancies and the tensions between, on the one hand, the knowledge and the skills of the teachers, and, on the other hand, the linguistic knowledge. The aim of our contribution is to revitalize teaching of verbs in school and to offer concrete didactic perspectives.

INDEX

Mots-clés : verbe, enseignants, pratiques, savoirs linguistiques, conjugaison

Keywords : verb, teachers, practices, linguistic knowledge, conjugation

\section{AUTEURS}

\section{PATRICE GOURDET}

Université de Cergy-Pontoise, F-95000, France

ESPÉ académie de Versailles

\section{MARIE-NOËLLE ROUBAUD}

Université Aix Marseille, ADEF, EA 4671, F-13100, France

ESPÉ académie d'Aix-Marseille 